\title{
Hyperfine interactions: the past, the present and the future
}

\author{
Guido Langouche
}

Published online: 19 August 2008

(C) Springer Science + Business Media B.V. 2008

\begin{abstract}
Five major hyperfine interaction techniques, detected by nuclear radiation, originated in the short time span between 1950 and 1965. The coincidence with the demographic expansion, especially in Europe, of university education led to the creation of many new research laboratories applying these promising techniques in solid state physics, chemistry and biology. Since the turn of century many of the early pioneers are going into retirement, leading to a decline in activities in Europe, compensated in some degree by an increase in activities outside Europe. The organisation of the $2007 \mathrm{HI} / \mathrm{NQI}$-conference was impeccable and took place in a superb setting. Thanks to all those involved in its organization.
\end{abstract}

Keywords Hyperfine interactions • Nuclear quadrupole interaction • Mössbauer spectroscopy • Perturbed angular correlations • Muon spin resonance • Nuclear orientation

I have divided my Concluding Remarks after the 2007 Hyperfine Interactions/ Nuclear Quadrupole Interactions Conference, held in Iguazú on the border between Argentina and Brazil, into three different paragraphs: the past, the present and the future.

\section{The past}

This is the 14th International Conference on Hyperfine Interactions. The series started in 1967 in Asilomar, CA, USA and was held since then every 3 years.

G. Langouche $(\bowtie)$

Physics Department, Institute of Nuclear and Radiation Physics,

Katholieke Universiteit Leuven, Leuven, Belgium

e-mail: guido.langouche@kuleuven.be 
The first conference was preceded, in the short period between 1950 and 1965, by a remarkable series of creations of five new hyperfine interactions techniques, all detected by nuclear radiation, following each other at an extremely rapid pace. Between 1950 and 1965 the scientific world witnessed first the creation of the perturbed angular correlation technique, which had to wait for the invention of the sodium iodide scintillation detector before it could measure the first g-factor reliably. This was followed in 1956 by the low temperature nuclear orientation technique, used by C.S. Wu to demonstrate parity violation in beta-decay, and less than 1 year later by the creation of the muon spin resonance technique in the demonstration of parity violation in the muon decay. Almost at the same time R. Mössbauer demonstrated the recoilless emission and absorption of gamma radiation by nuclei and created in this way Mössbauer spectroscopy, which was to become the most popular technique to measure hyperfine interactions at the site of the nucleus. The last nuclear hyperfine interaction technique in the series found its origin in the far east, where Sugimoto in Japan created the beta-nuclear magnetic resonance technique, using nuclei oriented in a nuclear reaction.

The creation of these five nuclear hyperfine interaction techniques was accidentally followed in this post-war period by a substantial demographic expansion of higher education in many countries, especially in Europe. Physics departments were expanding and young post-docs were appointed as new professors. Several of them had just learned of the possibilities to apply these new nuclear hyperfine techniques in nuclear physics, and more and more often also in solid state physics, in chemistry, in biology.

The annual publication output in hyperfine interactions kept rising until the mid 1980s. It then remained more or less constant until 2000. After 2000 statistics undoubtedly show a moderate decline in annual hyperfine interaction publication output. The same trend is observed in the participation rate in the International Hyperfine Interactions conferences. The fact that in 2004 the International Hyperfine Interaction conferences joined forced with the International Symposia in Nuclear Quadrupole Interactions, which are held jointly since then, could not change this trend.

Several reasons can be invoked to explain this tendency. The most obvious one is that many of the research groups created in the 1960s and 1970s are discontinued after the retirement of their laboratory leaders. Such an event is normally the occasion for any physics department to reflect on the type of physics research it wants to engage in the future through a new appointment. And the choice is often not hyperfine interactions. New experimental techniques to explore the fields of science of current interest have gained popularity, and "nuclear" is certainly not any more the fashion word it was half a century before.

Happily there are still quite a number of scientists who realize that the application of a hyperfine interaction technique, with its sub-nano sensitivity, to a modern scientific problem can allow for an original approach which can really make the difference. Occasionally rewarded by a publication in a top international journal.

\section{The present}

At every Hyperfine Interactions conference, the Concluding Remarks are the occasion for some statistics. Also here. The 118 participants are found to be spread over 
19 nationalities. A very unusual observation is that the majority of participants does not originate from one of the organizing countries. Brazil and Argentina each count 20 participants, but they are preceded by Japan, from where we count 21 delegates. Germany, the hyperfine interaction country by excellence, is a strong fourth with 17 participants. All other countries have sent participants in one digit numbers.

The organization was impeccable. Program and speakers of high level. The facilities excellent. The setting superb. Immediately after the preceding meeting, Bonn 2004, the organizers started working on the organization of this meeting and succeeded in providing a very smooth meeting. Chairman Alberto Pasquevich and his La Plata team at the Registration Desk (Laura, Claudia, Mario and all the others) did a great job. Supported, whenever necessary, by his Brazilian colleagues Helena Petrilli and Elisa Saitovich.

The organization of past Hyperfine Interaction conferences always included a substantial part of improvisation. Not any more. Starting with the present conference a well-defined structure of committees and guidelines guarding their functioning has been established. Bill Evenson, the chairman of the 2001 Hyperfine Interactions conference in Utah, and chairman of the Executive Committee, was instrumental in this. We thank him for this. Special thanks also to Ken-Ichi Kumagai, former chair of the NQI committee and to Gunther Schatz, who was one of the most active members of the Executive and Program Committees of so many Hyperfine Interactions conferences. They will be missed.

We welcome Reiner Vianden, the new chair of the Executive Committee, and Gary Wulfsberg, the new chair of the NQI committee.

\section{The future}

The venue of the $2010 \mathrm{HFI} / \mathrm{NQI}$ conference has been decided upon. It is somewhat unusual as it is neither a conference center nor a university. It is a world-renowned research center. The 2010 conference will be held in CERN, Geneva. It will be the occasion to get acquainted with the ISOLDE facility, where so many impressive hyperfine interactions studies have originated from in the last decades.

We hope to meet all of you again there. 